\title{
A revision of the fundamental parameters of the open cluster Hogg 15 and the projected star WR 47
}

\author{
A. E. Piatti ${ }^{1}$, E. Bica ${ }^{2}$, J. F. C. Santos Jr. ${ }^{3}$, and J. J. Clariá ${ }^{4}$ \\ 1 Instituto de Astronomía y Física del Espacio, CC 67, Suc. 28, 1428, Buenos Aires, Argentina \\ 2 Instituto de Fisica-UFRGS, CP 15051, CEP 91501-970 POA - RS, Brazil \\ 3 Departamento de Fisica, ICEx, UFMG, CP 702, 30123-970 BH - MG, Brazil \\ 4 Observatorio Astronómico, Universidad Nacional de Córdoba, Laprida 854, 5000 Córdoba, Argentina
}

Received 11 December 2001 / Accepted 1 March 2002

\begin{abstract}
We revise the fundamental parameters of the faint open cluster Hogg 15, for which two recent colourmagnitude diagram (CMD) studies have obtained significantly different ages. In the present study, we combine a series of methods trying to constrain age, together with other fundamental parameters. We employ spatial extractions to construct the CMDs, and the cluster integrated spectrum to compare it with those of templates of known age. We derive fundamental parameters, in particular, distance, of the closely projected Wolf-Rayet star HDE 311884 (WR 47) - often proposed to be physically related to Hogg 15. Based on the WR 47 spectrum and available photometry, we conclude that the short distance implied by the Hipparcos parallax (216 pc) is affected by binary motion. From the WR 47 spectrum we estimate a reddening $E(B-V)$ and a distance of $1.10 \pm 0.05$ and $5.2 \pm 0.9 \mathrm{kpc}$, respectively. For Hogg 15 we derive an age of $20 \pm 10 \mathrm{Myr}$, a reddening of $1.10 \pm 0.05$, and a distance of $3.1 \pm 0.5 \mathrm{kpc}$. We conclude that Hogg 15 is not related to WR 47 from the point of view of origin, since the cluster and the star do not belong to the same formation event.
\end{abstract}

Key words. techniques: photometric - techniques: spectroscopic - galaxy: open clusters and associations: general - galaxy: open clusters and associations: individual: Hogg 15 - stars: individual: WR

\section{Introduction}

Sometimes, independent investigations of individual open clusters yield different results which make it necessary to use additional tools to achieve greater precision. Frequently, disputed clusters are those embedded in crowded fields or highly reddened and/or poorly populated objects. For example, Westerlund (1987) and Piatti et al. (1998a) derived distances for Westerlund 1 of 5.0 and $1.0 \mathrm{kpc}$, respectively. M 73 (NGC 6994) is a case of a poorly populated object, possibly the remains of a 2-3 Gyr cluster (Bassino et al. 2000), but which has also been interpreted as a random field enhancement (Carraro 2000). Late stages in the cluster dynamical evolution might be the cause of several stellar concentrations which are present in open cluster catalogues, as pointed out by Bica et al. (2001) and Pavani et al. (2001). We conclude that open clusters whose fundamental properties are still under debate themselves constitute targets for new studies, especially with additional observational techniques.

Recently, Sagar et al. (2001, hereafter SMD) and Piatti \& Clariá (2001, hereafter PC) obtained multicolour CCD

Send offprint requests to: A. E. Piatti, e-mail: andres@iafe.uba.ar photometry for the southern open cluster Hogg 15. Despite the fact that their observations cover nearly $7 \mathrm{mag}$ of the cluster MS, age is a point of discrepancy between them. SMD conclude that the cluster, located at $3.0 \pm 0.3 \mathrm{kpc}$ from the Sun and affected by a non-uniform reddening with an average value of 1.15 , is very young, $6 \pm 2 \mathrm{Myr}$. On the other hand, PC find a slightly lower reddening, $E(B-V)=0.95 \pm 0.05$, a distance from the Sun of $2.6 \pm 0.8 \mathrm{kpc}$ and an age of $\sim 300 \mathrm{Myr}$. Previous photoelectric $U B V$ observations of 23 stars located in the cluster field by Moffat (1974) yielded colour excess $E(B-V)$, distance and age of $1.16 \mathrm{mag}, 4.2 \pm 0.4 \mathrm{kpc}$ and $\sim 8 \mathrm{Myr}$, respectively, whereas the matching of the cluster integrated spectrum with template spectra by Ahumada et al. (2000) led to $E(B-V)=1.05 \pm 0.05$ and an age of $5 \pm 2 \mathrm{Myr}$. Apparently, most of the results favour an extreme youth for Hogg 15, in agreement with the presence in the field of the bright Wolf-Rayet star HDE 311884 (WR 47). Indeed, although Ahumada et al. (2000) and SMD did not observe WR 47 - it is placed beyond what can be considered the cluster area by eye - they assumed its membership. Notice that PC did not observe WR 47 either and obtained an age $\sim 50$ times older. Thus, Hogg 15 calls for a careful revision of its fundamental parameters. 
In this paper, we revisit the data and analyses of PC and SMD, in search of a possible explanation for the origin of their discrepancies in the age of Hogg 15. A more complete handling of PC's photometric data and SMD results are presented in Sect. 2. In Sect. 3 we deal with the integrated spectrum obtained by Ahumada et al. (2000) rediscussed in terms of a new Galactic open cluster integrated spectral library. In Sect. 4 we discuss the WR 47 parameters. Finally, Sect. 5 summarises the main conclusions of this work.

\section{Photometric analysis of Hogg $\mathbf{1 5}$}

In this section, we consider the available data and information about Hogg 15, with a view to constraining the fundamental parameters.

\subsection{Piatti \& Clariá (2001)}

The $(V, B-V)$ and $(V, V-I)$ colour-magnitude diagrams (CMDs) obtained by PC (Fig. 5 of their paper) show a cluster MS with some particular features. Firstly, the cluster MS becomes broader and more populated from $V \sim 16$ until it reaches the photometry limit at $V \sim 20$ mag. Secondly, the upper MS is defined at its top by three bright stars $(V \sim 12)$ below which there is a gap of stars of roughly one magnitude. Thirdly, when matching the empirical isochrones of Piatti et al. (1998b) to the absolute $\left[M_{V},(V-I)_{\mathrm{o}}\right]$ plane (Fig. 10 of PC) possible distinct fiducial cluster sequences can be traced, each one following different curvatures and therefore having different Turnoff (TO) points. If we assign more weight to the lower MS, a $300 \mathrm{Myr}$ isochrone seems to be a reasonable representation of the cluster star distribution, the MS scatter and the unavoidable field star contamination being included. Precisely, this was the solution adopted by PC. However, the MS of Hogg 15 presents differences with respect to that of Mel 105, to which the authors attributed an age of $350 \mathrm{Myr}$ in the same work. If both clusters had comparable ages, the shape of their MSs should be similar. Both clusters present a curvature in the brighter parts of the MS/TO, though apparently less pronounced for Hogg 15, suggesting a younger age, but the field contamination hinders further inferences. In addition, Hogg 15 does not have red giant stars, which should be expected, since Mel 105 presents a well-defined giant clump. Lastly, from a structural point of view, Hogg 15 does not have the relaxed appearance of a $300 \mathrm{Myr}$ old cluster.

In order to clear up the age of Hogg 15, we first decided to improve its MS by removing the field star contamination as much as possible. To do this, we extracted from the data of $\mathrm{PC}$ those stars distributed within an ellipse centered at $\left(X_{\mathrm{c}}, Y_{\mathrm{c}}\right)=(380,230)$, and of semimajor and semi-minor axes of $80\left(36^{\prime \prime}\right)$ and $60\left(27^{\prime \prime}\right)$ pixels, respectively, as an attempt to define the cluster boundary. The resulting extracted absolute CMDs are shown in Fig. 1, where filled circles represent the stars encompassed by the ellipse. As can be seen, the cluster MS proves now to be better defined, and reflects the evolutionary stage of a younger open cluster than that determined by PC. With the aim of re-estimating its interstellar reddening, distance, and age, we fitted the cluster MS with the Zero Age Main Sequence (ZAMS) and isochrones computed by Lejeune \& Schaerer (2001). Adopting a solar metal content $(Z=0.02)$ for the cluster, we derived $E(B-V)=1.10 \pm 0.05, E(V-I)=1.40 \pm 0.05$, $m-M=16.0 \pm 0.2$ and $t=20 \pm 10$ Myr. Similar cluster parameters are obtained for adjacent metallicity values from the model grids $(Z=0.008$ or 0.04$)$. Figure 1 shows the placement of the ZAMS and the theoretical isochrones for $t=10,20$ and $50 \mathrm{Myr}$ superimposed as well. Notice that the MS/TO curvature neither corresponds to the family of cluster MSs with a few hundred million years, nor is as completely vertical as that of a 5 Myr old cluster. Therefore, the difference in the age of Hogg 15 between the present value and that of $\mathrm{PC}$ seems to come from the blurring of the MS/TO curvature mainly caused by the field star contamination.

We recall that the different parameter solutions obtained for Hogg 15 do not come from any kind of degeneracy in the isochrone sequence. Theoretical or empirical isochrones are not degenerate, since MS/TO are essencially all distinguishable in terms of shapes and curvatures. Degeneracy (blurring) for age solutions can arise from some of the following reasons, or combinations of them: (i) field contamination, (ii) differential reddening (iii) photometric errors and (iv) underpopulated clusters. Particularly, points (i) and (iv) play an important role in the estimation of the fundamental parameters of Hogg 15 from its CMDs. The estimated errors associated to the derived colour excesses and apparent distance modulus reflect the minimum colour and magnitude differences in the fitting of the ZAMS rather than the intrinsic dispersion of the cluster MS. From these values we derived a colour-to-colour $E(V-I) / E(B-V)$ ratio of $1.27 \pm 0.12$, which indicates that the interstellar absorption in the direction towards the cluster follows approximately the normal extinction law. Consequently, adopting the most frequently used value 3.2 for the total-to-selective extinction $R_{V}=A_{V} / E(B-V)$ ratio (Cousins 1978), we determined a cluster distance of $3.1 \pm 0.5 \mathrm{kpc}$. The distance error was computed with the expression: $\sigma(d)=0.46 \times[\sigma$ $\left.\left(V-M_{V}\right)+3.2 \times \sigma(E(B-V))\right] \times d$, where $\sigma\left(V-M_{V}\right)$ and $\sigma(E(B-V))$ represent the estimated errors in $V-M_{V}$ and $E(B-V)$, respectively. The resulting $E(B-V)$ colour excess is in agreement with previous estimations obtained by Moffat (1974), Ahumada et al. (2000), and SMD, whereas also the present cluster distance agrees with that of SMD.

\subsection{Sagar et al. (2001)}

SMD obtained $U B V R I$ CCD photometry for 337 stars in the field of Hogg 15. When comparing their photometry with that of PC for 122 stars in common, we find the following mean differences: $\Delta V_{\mathrm{SMD}-\mathrm{PC}}=-0.018 \pm 0.063$, 


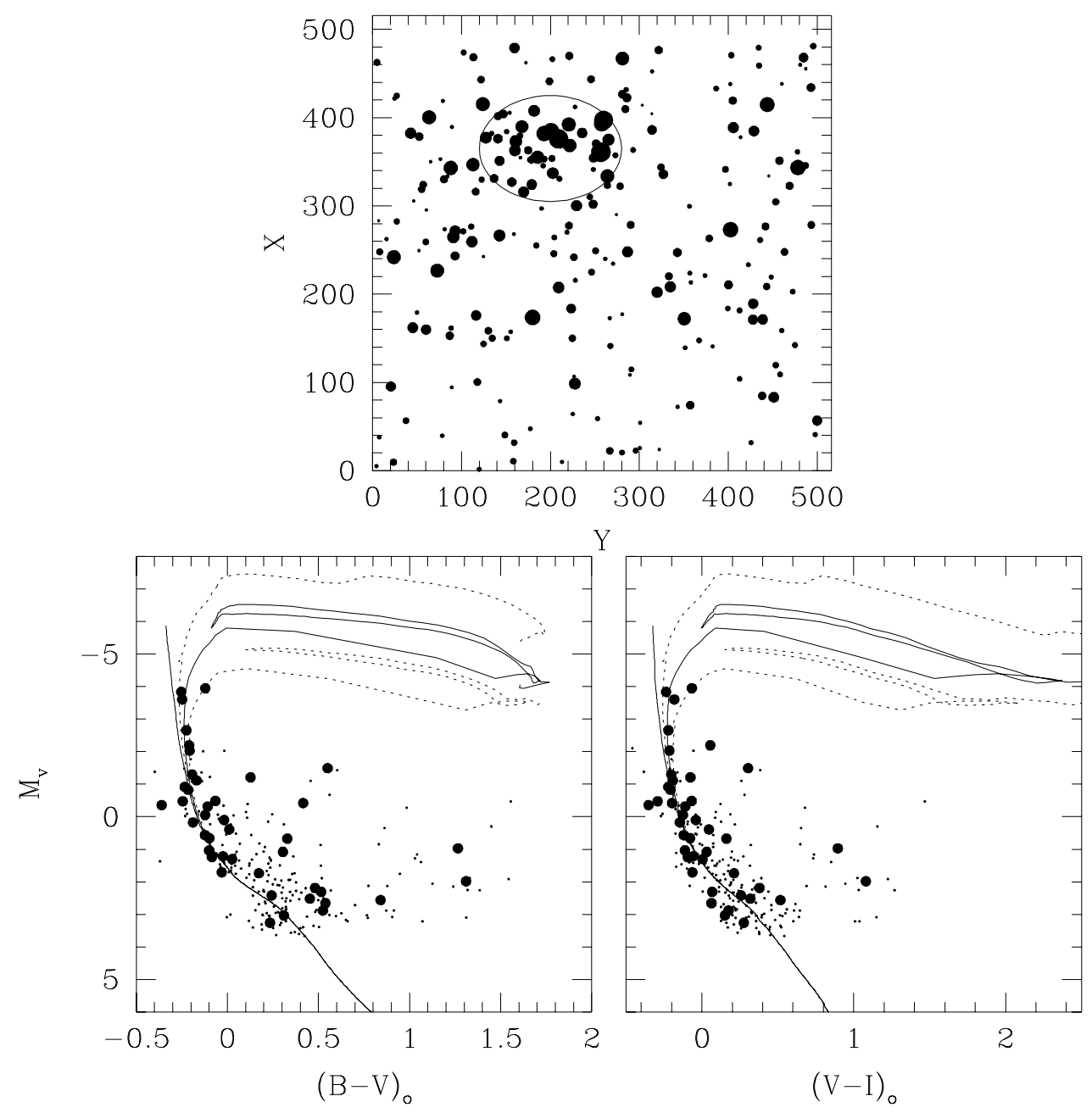

Fig. 1. Schematic finding chart and $\left[M_{V},(B-V)_{\mathrm{o}}\right]$ and $\left[M_{V},(V-I)_{\mathrm{o}}\right]$ diagrams of all the stars measured by Piatti \& Clariá (2001) in the field of Hogg 15. Filled circles in the absolute colour-magnitude diagrams correspond to stars distributed within the ellipse drawn in the schematic finding chart. The Zero Age Main Sequence and isochrones for $t=10,20$ and 50 Myr calculated by Lejeune \& Schaerer (2001) are also shown.

$\Delta(B-V)_{\mathrm{SMD}-\mathrm{PC}}=0.029 \pm 0.070$, and $\Delta(V-I)_{\mathrm{SMD}-\mathrm{PC}}=$ $0.135 \pm 0.102$. These differences suggest that both $V$ magnitude and $B-V$ colour values are within errors in the same system. This can also be seen in Fig. 2, where filled circles represent those stars distributed within the ellipse traced in Fig. 1. For $\Delta(V-I)_{\mathrm{SMD}-\mathrm{PC}}$ Fig. 2 shows a noticeable dependence with the magnitude for stars fainter than $V \sim 16$. In the present study, we will give more weight to the $(V, B-V)$ diagrams from both studies, but we would like to emphasize that the $V-I$ uncertainty on the fainter stars has hardly any effect on the interpretation of the brighter cluster sequences.

To estimate the cluster age from the fit of the observed cluster MS with theoretical isochrones, SMD assumed that the star WR 47 is a cluster member. Consequently, WR 47 had an important weight in the SMD derivation of a very young age for the cluster. However, WR 47 lies outside the cluster radius estimated by Moffat (1974) (see his Fig. 1) at a distance where the cluster star density drops off more than 2.5 times the central cluster star density, just close to the background star level (see Fig. 15 of SMD). The star is a binary (see Sect. 4) and, as such, it is much brighter than any field star observed towards Hogg 15 . Its $V$ magnitude and $U-B$ and $V-B$ colours were taken from Moffat (1974).

\section{Spectroscopic analysis}

Ahumada et al. (2000) performed estimates of age and foreground reddening by comparing the continuum distribution and line strengths of the Hogg 15 flux-calibrated integrated spectrum with those of template cluster spectra with known parameters. They derived a colour excess $E(B-V)$ and an age of $1.05 \pm 0.05$ and $5 \pm 2 \mathrm{Myr}$, respectively, from matching the object spectrum to that of the template spectrum which most resembles it. They also determined an age of $30 \mathrm{Myr}$ from the Balmer line equivalent widths (Bica \& Alloin 1986a,b). However, they preferred the younger age since it was compatible with the 


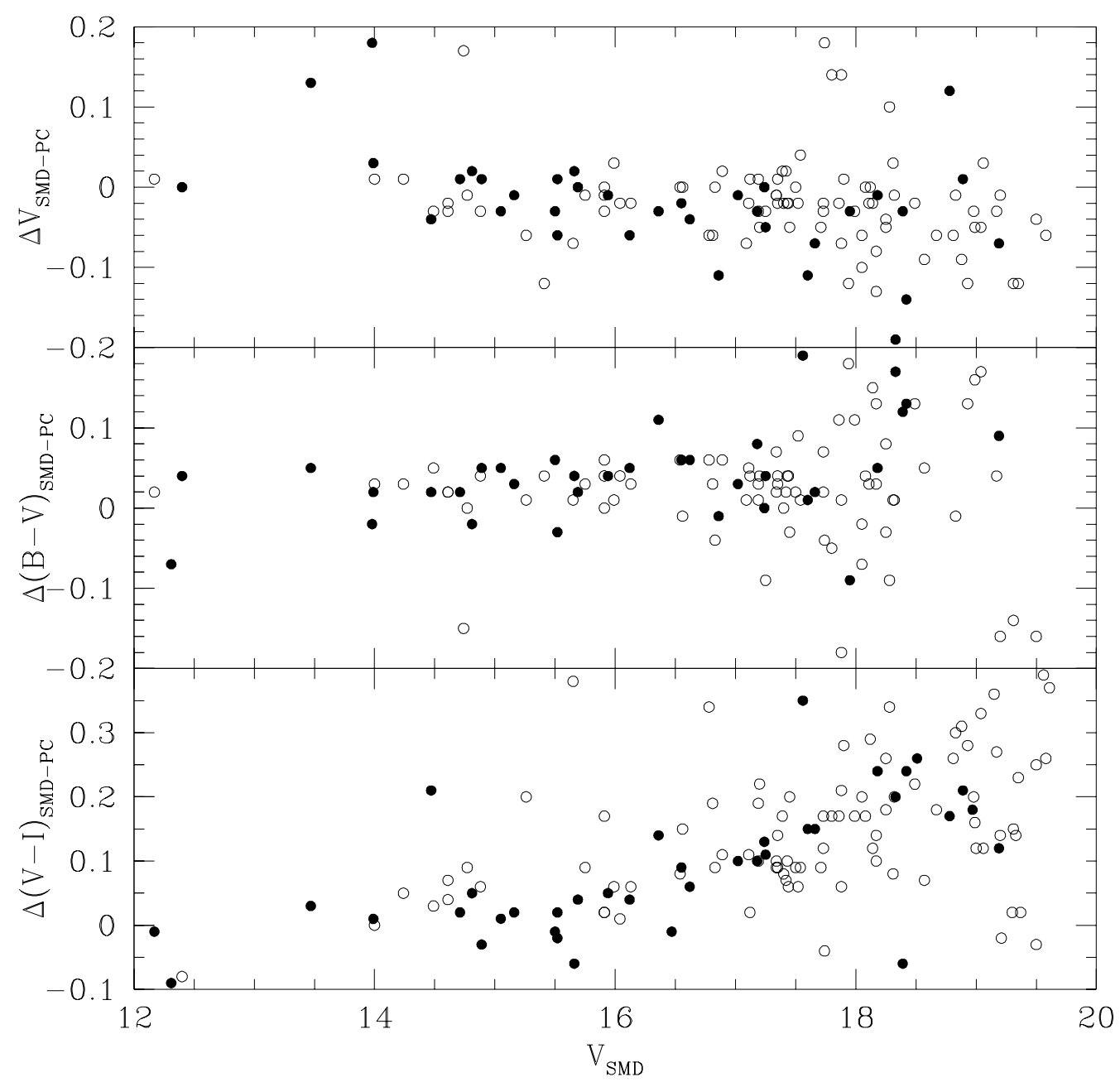

Fig. 2. Magnitude and colour differences between Sagar et al. (2001, SDM) and Piatti \& Clariá (2001, PC) for Hogg 15 as a function of $V_{\mathrm{SMD}}$. Filled circles represent stars distributed within the ellipse drawn in Fig. 1.

assumed membership of WR 47. Additionally, with the set of template spectra used by Ahumada et al. (2000), the representation of clusters' evolution is quite detailed from 3 up to $9 \mathrm{Myr}$, but of a lower resolution for older stages.

As a consequence, we decided to build an updated template spectra library using the Galactic cluster integrated spectra available in the literature so far. The new set of template spectra has the advantage of being defined for solar metal content, in contrast with previous spectral libraries based mostly on integrated spectra of Magellanic Cloud clusters for the age range relevant to the present study (Bica 1988; Santos et al. 1995). The library, based on nearly 50 cluster spectra, basically covers the whole age range of the Galactic open clusters with an improved age resolution. We will be describing details on the construction of this library in a forthcoming paper. The present study, however, will focus exclusively on the use of the resulting template spectra to estimate the age of Hogg 15 . Figure 3 shows two reddening corrected integrated spectra of Hogg 15 and the new template spectra of 4-8 and 20 Myr superimposed. The cluster spectra are corrected for the amount of reddening so as to produce the best match of the template spectra. All the spectra were normalized at $\lambda=5870 \AA$ and shifted by different constants for the sake of comparison. As can be seen, only the $20 \mathrm{Myr}$ template spectrum clearly reproduces the cluster spectral features along the whole observed spectral range. The most noticeable difference between both template spectra is a poor match around $4800 \AA$ for the $4-8$ Myr template. This result confirms the hypothesis anticipated in Sect. 2.1.1, that Hogg 15 is neither a 5 Myr old cluster nor a 300 Myr old one, the latter having much deeper Balmer lines and a shallower continuum energy distribution than those observed in the cluster spectrum.

The above result shows that the integrated spectroscopy is an important tool for obtaining reddening estimates and ages not only of small and compact star clusters (see, e.g., Bica et al. 1995, 1998) but also of underpopulated objects, owing to the information contained in the spectral lines. In addition, this technique is definitely suitable to remove the MS/TO degeneracy in CMDs of relatively young clusters caused by the field star contamination. For additional age constraints, we also measured 


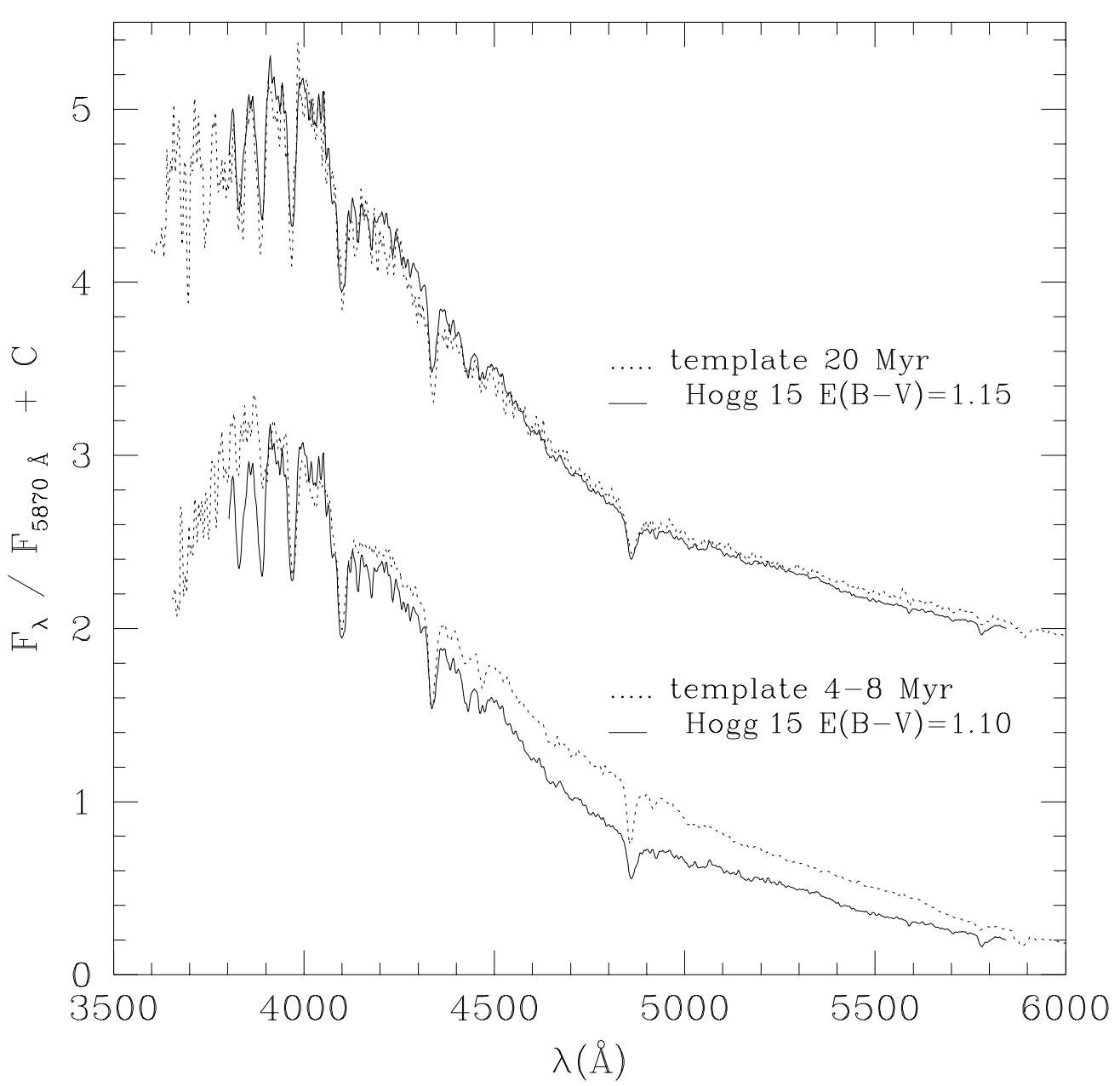

Fig. 3. Reddening-corrected integrated spectra of Hogg 15 and the 4-8 and 20 Myr template spectra superimposed. Spectra are in relative $F_{\lambda}$ units normalized at $5870 \AA$. Constants have been added to the spectra for comparison purposes.

the equivalent widths of the Balmer lines using IRAF $^{1}$ tasks contained in the ONEDSPEC package and found a value of $20 \pm 10 \mathrm{Myr}$.

WR 47 is projected at $\approx 2^{\prime}$ east of the Hogg 15 center. Since the integrated spectrum was scanned in the northsouth direction, the WR star was sampled near the edge of the spectrograph slit which corresponds to a length of $4.7^{\prime}$ on the sky and not included in the cluster integrated spectrum extraction. Massive stars, such as WR stars, which are sure cluster members are often found in the main body of their clusters, as in NGC 3603, NGC 6231 (Santos \& Bica 1993) and Westerlund 2 (Piatti et al. 1998a), where the WR features are evident in the integrated spectra.

\section{WR 47 revisited}

Since the membership issue of HDE 311884 has been used as a constraint for estimating the age of Hogg 15, we further discuss its fundamental parameters.

\footnotetext{
${ }^{1}$ IRAF is distributed by the National Optical Astronomy Observatories, which is operated by the Association of Universities for Research in Astronomy, Inc., under contract with the National Science Foundation.
}

HDE 311884 (WR 47, LSS 2745, HIP 62115) is classified as a $\mathrm{WN} 6+\mathrm{O} 5 \mathrm{~V}$ on the basis of its spectrum (e.g., van der Hucht 2001). According to its light curve (Moffat et al. 1990), the orbit period is $\approx 6.2$ days and the binary components have $M_{V}(\mathrm{WN} 6)=-5.2, M_{V}(\mathrm{O} 5 \mathrm{~V})=-5.7$, giving a relative brightness $I_{V}(\mathrm{WN} 6) / I_{V}(\mathrm{O} 5 \mathrm{~V})$ of 0.64 (Lamontagne et al. 1996). The resulting combined absolute magnitude is thus $M_{V}=-6.2 \pm 0.2$, which makes WR 47 the brightest star not only in the cluster area but also in the field surrounding the cluster. The uncertainty in the absolute magnitude $M_{V}$ was estimated from the difference between the present value and that obtained by Lundström \& Stenholm (1984).

Concerning its distance, the Hipparcos parallax (4.63土 2.01 mas) yields $216_{-65}^{+166} \mathrm{pc}$, well below the photometric value for the cluster. This distance would make the star the closest WR, just behind or inside the Coalsack dark nebula, which is recognizably not associated with any star formation activity. However, the Hipparcos parallaxes produce distances with biases which increase with the relative error $\sigma_{\pi} / \pi$ (e.g., Luri \& Arenou 1997). In addition, binarity can also affect the measured parallax. Indeed, it has been argued by Pozzo et al. (2000) that $\gamma^{2}$ Vel (HD 68273, WR 11), another WR star in a binary system, is at 


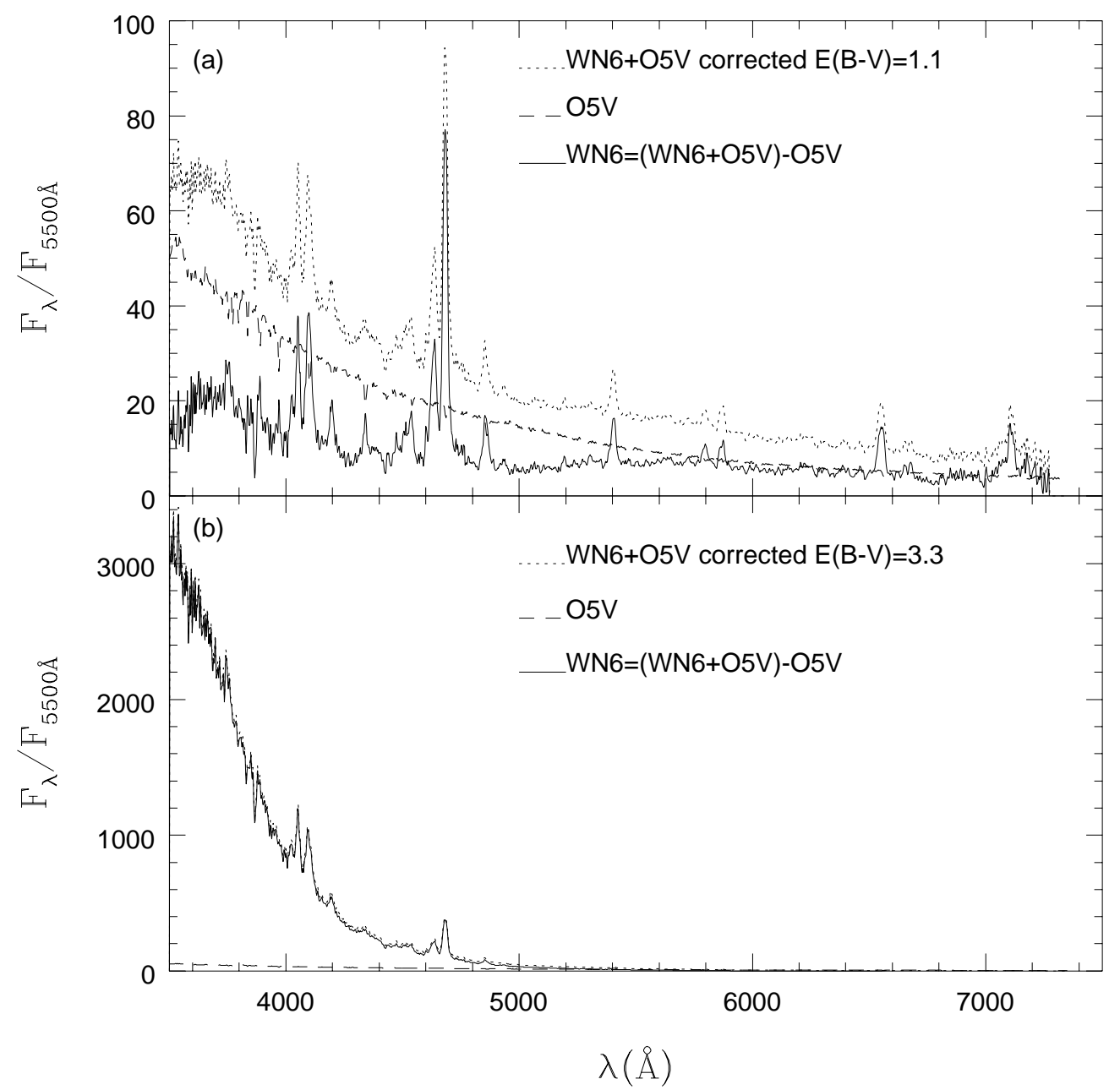

Fig. 4. Spectral decomposition of HDE 311884 (WR 47) into its components. The binary observed spectrum is corrected for a foreground reddening of a) $E(B-V)=1.1$ and $\mathbf{b}) E(B-V)=3.3$. The pure WN6 spectrum results from the subtraction of the $\mathrm{O} 5 \mathrm{~V}$ spectrum from the binary spectrum. We used as weights the relative flux fraction at $5500 \AA$, as given by the absolute magnitudes in Lamontagne et al. (1996): $F_{V}(\mathrm{WN} 6+\mathrm{O} 5 \mathrm{~V})=16.4$ and $F_{V}(\mathrm{O} 5 \mathrm{~V})=10$.

a distance of $425 \pm 65 \mathrm{pc}$, as derived by photometric means, and not at $258_{-31}^{+41} \mathrm{pc}$, according to Hipparcos. In both cases, the distances are underestimated when obtained from the Hipparcos parallaxes. If WR 47 is located nearby the Coalsack, it must be significantly reddened. By using $V=10.89, M_{V}=-6.2$ and $d=216 \mathrm{pc}$, the resulting colour excess turns out to be $E(B-V)=3.3$, i.e., three times higher than the estimated average reddening for the cluster.

In order to assess empirically the reddening effect on the spectral distribution of WR 47, we have employed the spectrophotometric catalogue of Wolf-Rayet stars by Torres-Dodgen \& Massey (1988, available at CDS), which contains optical observations at $10 \AA$ resolution for 173 WR stars, including WR 47. Figure 4 is a simple decomposition of WR 47 into two components. In Fig. 4a the observed spectrum is corrected for a reddening of $E(B-V)=1.1$ and subtracted from a $05 \mathrm{~V}$ type spectrum (taken from the Jacoby et al. 1984 spectral library) in order to give a WN6 pure spectrum. The adopted weights for each spectrum at $5500 \AA$ follow the absolute $V$ brightness ratio according to Lamontagne et al. (1996). The same procedure is applied to Fig. $4 \mathrm{~b}$ for $E(B-V)=3.3$. Clearly, the resulting WN6 spectrum is completely different in each case. The reddening effect on the composite $\mathrm{WN} 6+\mathrm{O} 5 \mathrm{~V}$ observed spectrum of WR 47 is presented in Fig. 5 for progressive $E(B-V)$ corrections. Finally, Fig. 6 compares ten similar type WR spectra (including WR 47) taken from Torres-Dodgen \& Massey (1988) and reddening corrected according to the $E(b-v)$ value provided by van der Hucht (2001). We have used the relation $E(B-V)=1.21 E(b-v)$ (Lundström \& Stenholm 1984). An exception was made with WR 47 which has been corrected by $E(B-V)=1.1$, different from the value of 1.17 derived from the relation of Lundström \& Stenholm (1984). The spectral types quoted are also from van der Hucht (2001). As can be seen, WR 47 does not appear to be affected by an interstellar reddening much higher than that associated to Hogg 15 , namely $E(B-V)=1.1$. We found that an error of 0.15 in $E(B-V)$ yields a change of $\approx 22 \%$ in the flux of the WR 47 spectrum at $3800 \AA$, significantly displacing it from the mean spectral energy distribution of similar type WRs in 


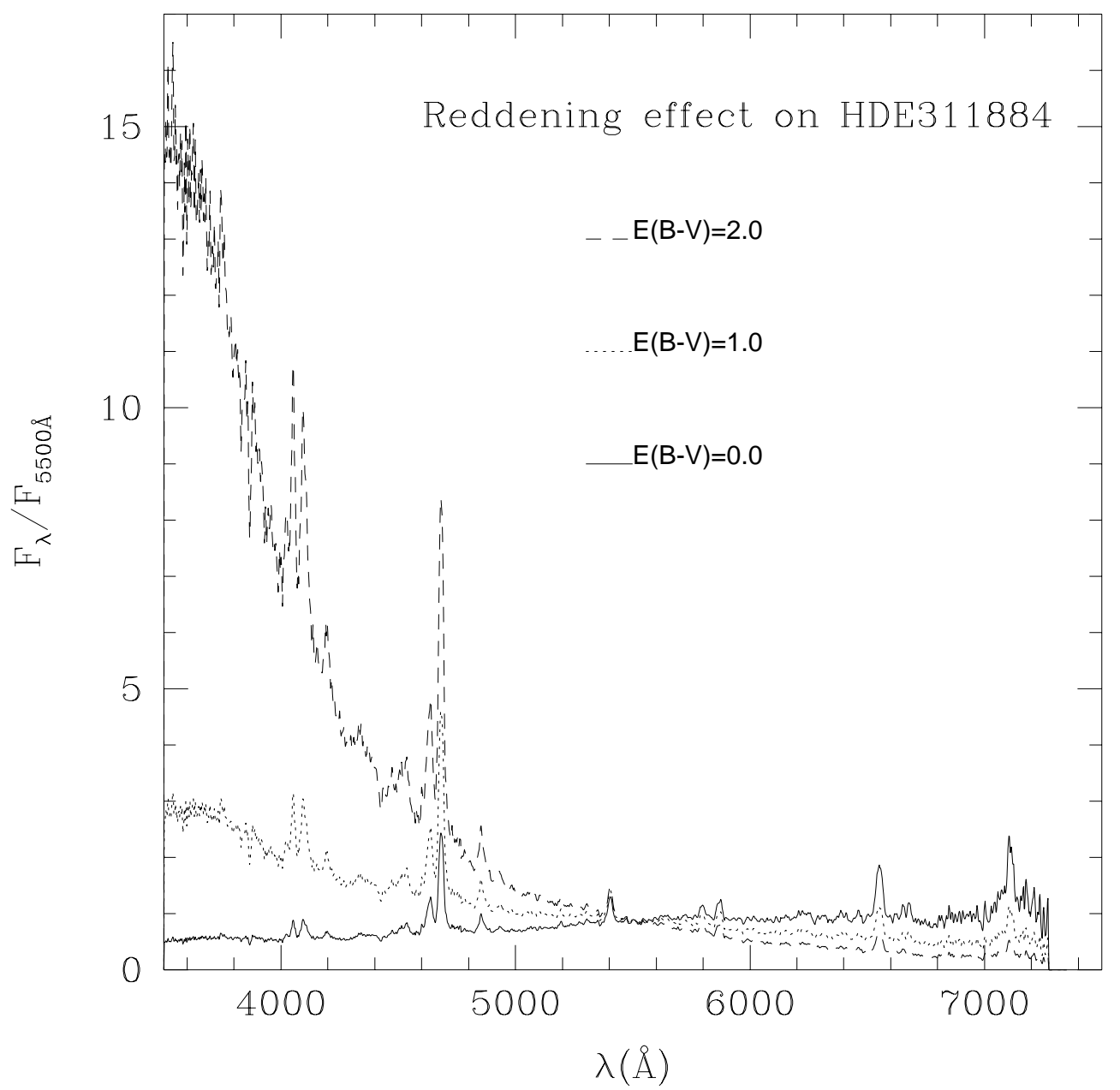

Fig. 5. Progressive reddening corrections spanning $E(B-V)=2.0$ are applied to the spectral energy distribution of HDE 311884 (WR 47) showing the significant changes on its slope.

the blue. An error of 0.10 in $E(B-V)$ yields a change of $15 \%$ at the same wavelength and also causes a conspicuous difference of the WR 47 spectrum with respect to the mean spectrum. On the contrary, around $8 \%$ in flux at $3800 \AA$ is the change yielded by an error of 0.05 in $E(B-V)$, the WR 47 spectrum bearing a resemblance to those of a similar type. In this respect, we rule out any large systematic deviation in the reddening values published by van der Hucht (2001), since they are based on several previous independent studies.

Assuming that the star is reddened by $E(B-V)=$ $1.10 \pm 0.05$, and using the apparent magnitude $V(\mathrm{WN} 6+\mathrm{O} 5 \mathrm{~V})=10.89$ measured by Moffat (1974) and the absolute magnitude $M_{V}=-6.2$ derived above, its resulting distance is $5.2 \pm 0.9 \mathrm{kpc}$, approximately $2 \mathrm{kpc}$ further from the Sun than Hogg 15. The distance error was computed with the expression: $\sigma(d)=0.46 \times$ $\left[\sigma V+\sigma M_{V}+3.2 \times \sigma(E(B-V))\right] \times d$, where $\sigma V(=0.018)$ and $\sigma M_{V}(=0.2)$ are in agreement with Moffat (1974) and Lundström \& Stenholm (1984), respectively. van der Hucht (2001) estimated a distance of $3.8 \mathrm{kpc}$ for WR 47 based on the narrow-band photometric system of Smith (1968) primarily designed for spectral classifications of WRs. Indeed, some of the five bands include WR emission lines for such purpose, and they might disturb distance estimates from such narrow bands. Besides, the resulting comparable reddening between Hogg 15 and WR 47 for differing distances might be explained in terms of depletion of dust in that path. Variations in terms of depletion and excess of dust are not unexpected in the interstellar medium. The interception of discrete dust clouds and bubbles arising as a consequence of massive star evolution (dust sweeping by winds and dust destruction by UV photons) may be responsible for deviations from a normal distance/reddening relation.

The issue of whether WR 47 is associated with Hogg 15 or not now appears to be clarified. WR 47 and Hogg 15 share the same reddening, a fact probably mostly caused by being both affected by the Coalsack cloud. Even so, the resulting distance for WR 47 largely exceeds that of the cluster, and if WR 47 were placed at the Hipparcos' parallax, it would be affected by an interstellar reddening 3 times larger than that for the cluster. Both reddening and distance values cannot be adjusted to the cluster values simultaneously.

\section{Concluding remarks}

The origin of the current investigation lies mainly in the noticeable discrepancies, particularly in age, found by 


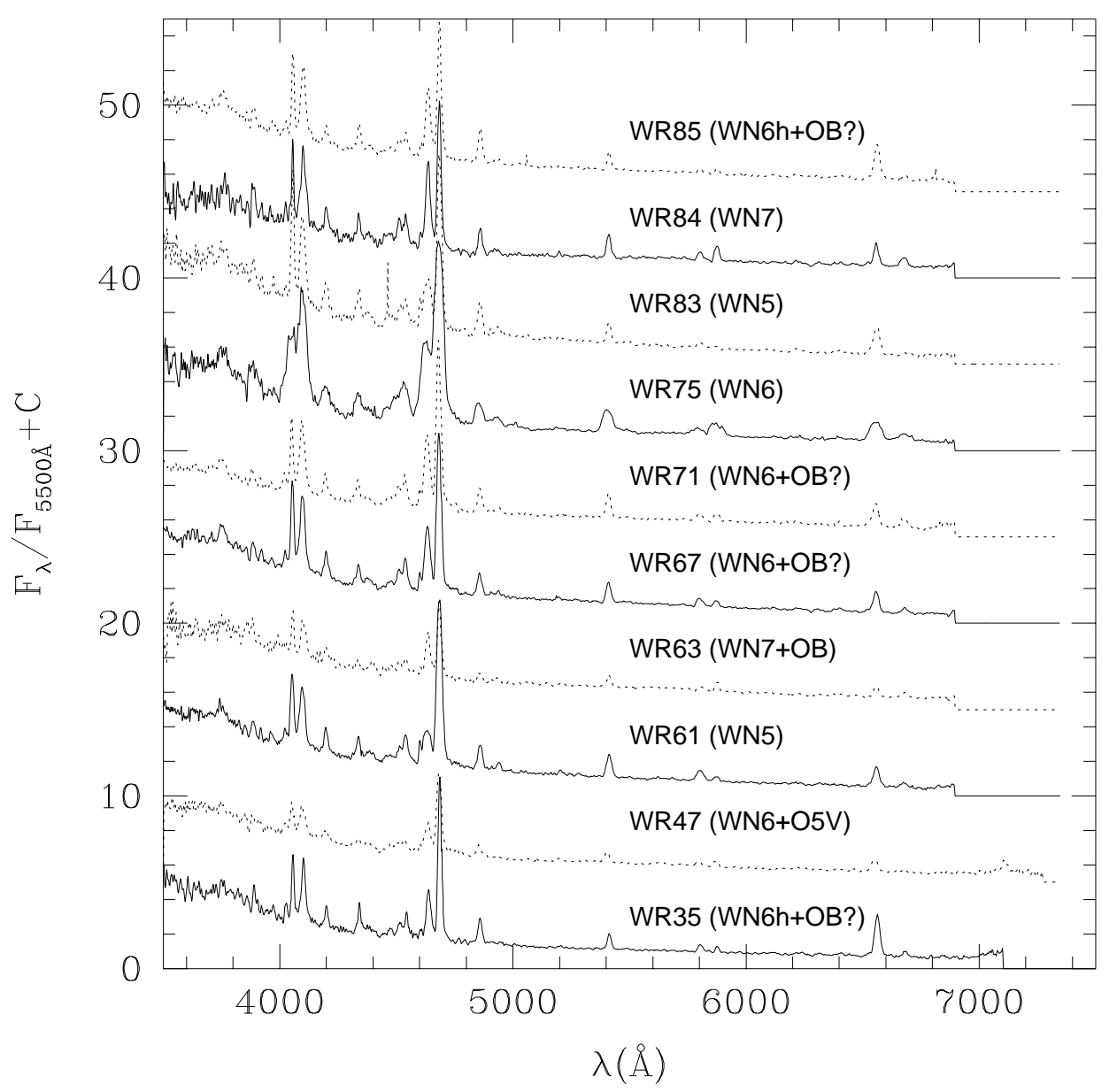

Fig. 6. A comparison of WR stars of similar types. The spectra are the observed ones from Torres-Dodgen \& Massey (1988) corrected for $E(B-V)$ according to van der Hucht (2001), except WR 47 spectrum, for which $E(B-V)=1.1$ was used. The spectra are normalized at $5500 \AA$ and shifted by a constant.

Piatti \& Clariá (2001, PC) and Sagar et al. (2001, SMD) in very recent photometric studies of the southern open cluster Hogg 15. More precisely, while based on CCD BVI photometry down to $V \sim 19 \mathrm{mag}, \mathrm{PC}$ estimate the age of this object to be $\sim 300 \mathrm{Myr}$, SMD derive a much younger age of $6 \pm 2 \mathrm{Myr}$, based on CCD $U B V R I$ data down to $V \sim 20$ mag. Even if $V$ magnitudes and $B-V$ colours show good agreement between both investigations, the $V-I$ colours for stars fainter than $V \sim 16$ mag do not. These discrepancies, however, do not interfere with the interpretation of the brighter cluster sequences, and therefore, do not account for the significant differences found in age. Clearly, the much younger age obtained by SMD is mainly due to the assumed cluster membership of the closely projected Wolf-Rayet star HD 311884 (WR 47).

We conclude that the estimation of an older age, in PC's study, can be accounted for in terms of the MS/TO curvature being blurred in the CMDs, an effect mainly caused by field star contamination. We employed spatial extractions for the CMDs in order to define the cluster MS more precisely, using PC's data. The resulting cluster MS exhibits now an MS/TO curvature which corresponds neither to those typical of open clusters of a few hundred million years nor to a 5 or 6 Myr cluster, for which we would expect an almost completely vertical MS. Fitting the now better-defined cluster MS with the ZAMS and isochrones by Lejeune \& Schaerer (2001), we derived $E(B-V)=1.10 \pm 0.05, E(V-I)=1.40 \pm 0.05$, $m-M=16.0$ - equivalent to $3.1 \pm 0.5 \mathrm{kpc}$ if $\mathrm{R}_{V}=$ $\mathrm{A}_{V} / E(B-V)=3.2$ is adopted - and an age of $20 \pm 10 \mathrm{Myr}$ for $\operatorname{Hogg} 15$.

On the other hand, Ahumada et al. (2000) estimated the age of Hogg 15 as $5 \pm 2 \mathrm{Myr}$ and $\sim 30 \mathrm{Myr}$ from matching the cluster integrated spectrum to that of the template spectrum which most resembles it and from the Balmer line equivalent widths, respectively, but they adopted the younger age because it is compatible with the assumed membership of WR 47. However, if a new set of template spectra defined for solar metal content with an improved age resolution is used, then the 20 Myr template spectrum clearly reproduces the cluster spectral features along the whole observed spectral range, the resulting $E(B-V)$ value being practically unaltered. For further age constraints, we also measured the equivalent widths of the 
Balmer lines in the integrated cluster spectrum, obtaining a value of $20 \pm 10 \mathrm{Myr}$. The preceding results lead to the conclusion that the integrated spectroscopic technique is of great value as an instrument of age estimation, equally suitable for small and compact star clusters and for underpopulated objects as well. The technique is also particularly useful to remove the MS/TO degeneracy, generated by field star contamination in CMDs of comparatively young clusters.

To clarify the issue of whether the Wolf-Rayet star HDE 311884 (WR 47), known to be a WN6+O5V binary system, is associated or not with $\operatorname{Hogg} 15$, we discuss its fundamental parameters, particularly its distance. Based on the WR 47 spectrum and available photoelectric photometry, we assume that the underestimated distance implied by the Hipparcos parallax (216 pc) is affected by its binary character. By comparing the WR 47 spectrum with those of WR stars of a similar type, we conclude that WR 47 is not affected by a $E(B-V)$ colour excess much higher than that associated with Hogg 15, namely $E(B-V)=1.10$. On the basis of WR 47's resulting distance $(5.2 \pm 0.9 \mathrm{kpc})$ by large surpassing that of the cluster, we sustain that WR 47 is not physically related to Hogg 15.

Acknowledgements. We gratefully acknowledge financial support from the Brazilian institutions CNPq and FINEP, and the Argentinian institutions CONICET, SECYT (Universidad Nacional de Córdoba), and AGENCIA CÓRDOBA CIENCIA. We also thank the referee J. C. Mermilliod for his comments and suggestions. This research has made use of the VizieR catalogue access tool, and of the SIMBAD database, operated at CDS, Strasbourg, France.

\section{References}

Ahumada, A. V., Clariá, J. J., Bica, E., \& Piatti, A. E. 2000, A\&AS, 141, 79

Bassino, L. P., Waldhausen, S., \& Martínez, R. E. 2000, A\&A, 351,138
Bica, E. 1988, A\&A, 195, 76

Bica, E., \& Alloin, D. 1986a, A\&A, 66, 171

Bica, E., \& Alloin, D. 1986b, A\&AS, 162, 21

Bica, E., Clariá, J. J., Bonatto, Ch., et al. 1995, A\&A, 303, 747

Bica, E., Clariá, J. J., Piatti, A. E., et al. 1998, A\&AS, 131, 483

Bica, E., Santiago, X., Dutra, C. M., et al. 2001, A\&A, 366, 827

Carraro, G. 2000, A\&A, 357, 145

Cousins, A. W. J. 1978, Mon. Not. R. Astron. Soc. S. Afr., 37,62

Jacoby, G. H., Hunter, D. A., \& Christian, C. A. 1984, ApJS, 56,257

Lamontagne, R., Moffat, A. F. J., Drissen, L., et al. 1996, AJ, 112,2227

Lejeune, T., \& Schaerer, D. 2001, A\&A, 366, 538

Luri, X., \& Arenou, F. 1997, Proceedings of the ESA Symposium Hipparcos - Venice 1997, 13-16 May, Venice, Italy, ESA SP-402, 449

Lundström, I., \& Stenholm, B. 1984, A\&AS, 58, 163

Moffat, A. F. J. 1974, A\&A, 34, 29

Moffat, A. F. J., Drissen, L., Robert, C., et al. 1990, ApJ, 350, 767

Pavani, D. B., Bica, E., Dutra, C. M., et al. 2001, A\&A, 374, 554

Piatti, A. E., \& Clariá, J. J. 2001, A\&A, 370, 931 (PC)

Piatti, A. E., Bica, E., \& Clariá, J. J. 1998a, A\&AS, 127, 423

Piatti, A. E., Clariá, J. J., \& Bica, E. 1998b, ApJS, 116, 263

Pozzo, M., Jeffries, R. D., Naylor, T., et al. 2000, MNRAS, 313,23

Sagar, R., Munari, U., \& de Boer, K. S. 2001, MNRAS, 327, 23 (SMD)

Santos, J. F. C. Jr., \& Bica, E. 1993, MNRAS, 260, 915

Santos, J. F. C. Jr., Bica, E., Clariá, J. J., et al. 1995, MNRAS, 276,1155

Smith, L. F. 1968, MNRAS, 140, 409

Torres-Dodgen, A. V., \& Massey, P. 1988, AJ, 96, 1076

van der Hucht, K. A. 2001, New Astr. Rev., 45, 135

Westerlund, B. E. 1987, A\&AS, 70, 311 